\title{
Mesenchymal Stem Cells for the Treatment of Intestinal Ischemia-reperfusion Injury: a Protocol of Systematic Review and Meta-analysis of Animal Models
}

\section{Guangru Zhang}

Lanzhou University https://orcid.org/0000-0002-5875-7642

\section{Shenghong Suo}

Qinghai Institute of Health Sciences

Zhenzhen Liu

Lanzhou University

Disheng Liu

Lanzhou University

\section{Zhiyu Zhao}

Lanzhou University

\section{Long Ge}

Lanzhou University

\section{Yufang Leng ( $\boldsymbol{\nabla}$ lengyf_Izu@126.com )}

Department of First Clinical Medical College, Lanzhou University https://orcid.org/0000-0001-70942413

\section{Protocol}

Keywords: Intestinal ischemia-reperfusion (I/R), Mesenchymal stem cells (MSCs), injury, animal models, protocol, systematic review

Posted Date: June 9th, 2021

DOI: https://doi.org/10.21203/rs.3.rs-582832/v1

License: (c) (i) This work is licensed under a Creative Commons Attribution 4.0 International License. Read Full License 


\section{Abstract}

Introduction: Intestinal ischemia-reperfusion (I/R) injury is a common clinical event. Mesenchymal stem cells (MSCs) have been widely used to repair intestinal injury in animal models. However, the effects of MSCs on intestinal I/R injury therapy remain unclear. Thus, we will perform a systematic review and meta-analysis of controlled trials to evaluate the efficacy of MSCs in animal models of intestinal I/R injury.

Methods and analysis: We will search PubMed, Web of Science, Embase, Cochrane Library, Science Citation Index, China National Knowledge Information database, Wanfang Database, and the Chinese Scientific and Technological Journal Database in May 2021. We will include studies that evaluate the two different interventions for target MSCs to be maintained for the degree of histopathologic changes, mortality rate of rats, tumour necrosis factor $a$, and diamine oxidase. Two reviewers will independently screen titles and abstracts, perform a full article review, and extract study data. We will also use the SYRCLE tool to assess the risk of bias in the included studies. Furthermore, a random-effects metaanalysis will be conducted. Dichotomous and continuous outcomes will be analysed using risk ratios with 95\% confidence intervals (Cls) and weighted mean difference with 95\% Cls, respectively. For outcomes where different scales or different measurement methods have been used, the standardised mean difference will be applied. Subgroup and sensitivity analyses will be performed to explore the heterogeneity. Stata (version 12.0, Stata Corp, College Station, Texas, USA) will be used to analyse and pool the individual research results.

Ethics and dissemination: This systematic review and meta-analysis does not require an ethical approval because no human beings are involved. We aim to publish this systematic review in a peer-reviewed journal.

PROSPERO registration number: CRD42021231826

\section{Strengths And Limitations Of This Study}

- This systematic review will be the latest study to determine the efficacy of MSCs in animal models of intestinal I/R injury.

- This systematic review will be performed with rigorous study screening, data extraction, and risk of bias assessment.

- The expected major limitations of this review will be the clinical heterogeneity of the included trials and the diversity of the MSCs.

- The number of studies with eligible data for subgroup analyses and publication bias may be limited.

\section{Introduction}


Intestinal ischemia-reperfusion (I/R) injury is a common (1/1000 hospital admissions) and serious clinical event, which can be caused by different pathophysiological factors such as intestinal obstruction, volvulus, superior mesenteric artery embolism, haemorrhagic shock, severe trauma, and intestinal transplantation[1, 2]. The intestinal tissue is the largest reservoir of bacteria in the body. When ischemia occurs, the intestinal tissue is in a state of ischemia and hypoxia, and the energy metabolism and morphological structure of cells are seriously damaged; when the blood supply is restored, the intestinal bacteria shift, and the endotoxin carried by the bacteria enters the peripheral blood circulation, causing serious damage to the endothelial cells that could lead to acute inflammation and other reactions. In severe cases, it can cause inflammatory reactions in local and distant organs (such as the lungs [3] and liver [4]), promote the occurrence and development of multiple organ dysfunction syndrome, and lead to a variety of perioperative complications and high mortality $[5,6]$. Due to delayed diagnosis and lack of efficient treatment, it is reported that the mortality of acute mesenteric ischemia patients is as high as $60-80 \%[7]$. Another study found that intestinal I/R injury is becoming the biggest obstacle to improving the outcome of intestinal transplantation[8]. Studies on the mechanism of intestinal I/R injury have shown that its occurrence is mainly related to oxygen free radical injury[9], calcium overload[10], inflammatory cytokine release[11], and apoptosis[12]. Although the mechanism has been studied indepth, treatments for intestinal I/R injury, including nitric oxide supplementation, antioxidants, anticomplement therapy, free-radical scavengers, anti-leukocyte therapy, glutamine supplementation, and glycine supplementation, are still inadequate[13].

Stem cell therapy has become a new strategy for the repair of various ischemia and reperfusion injury diseases[14]. As one of the most popular pluripotent stem cells, mesenchymal stem cells (MSCs) have been widely studied in the past decades[15]. MSCs are multipotent cells with low immunogenicity and immunoregulation[16-18], and can be easily isolated and expanded from the bone marrow and other tissues, including the placenta, amniotic fluid, umbilical cord tissues, adipose tissue, testis, or lungs[19, 20]. It has been reported that when tissue and organ damage occurs, MSCs can be transplanted into injured tissues to play a role in repair through differentiation and replacement of damaged cells[21, 22]. Furthermore, MSCs can also secrete many protective factors, such as the epithelial growth factor, vascular endothelial growth factor, transforming growth factors $\alpha$ and $\beta$, fibroblast growth factor, insulinlike growth factor type 1, to exert a protective effect through paracrine function[23, 24]. Several studies have confirmed that MSCs can repair the injury of many tissues and organs such as myocardial infarction[25], ischemic brain injury[26], spinal cord injury[27], and liver[28] and kidney [29] I/R injuries. Therefore, MSCs have the potential for application in a wide variety of degenerative disorders.

The first animal experiment to investigate MSCs for the treatment of intestinal I/R injury was conducted in 2009 and reported that the local administration of bone marrow-derived MSCs (BM-MSCs) can alleviate intestinal I/R injury in rats[12]. Subsequently, many studies on the effects of MSCs on animal models of intestinal I/R injury have been published. Shen et al. reported that BM-MSCs can reduce intestinal I/R injury in rats via a TNF-a-regulated mechanism[30]. Additionally, Jiang et al. found that BMMSCs not only inhibit the release of proinflammatory cytokines and suppress the overexpression of proinflammatory genes, but also accelerate the expression of proliferative genes involved in intestinal 
mucosal cellular regeneration[31]. MSCs can also protect against intestinal I/R injury by increasing the antioxidant capacity of small bowel tissues after the injury[32]. In addition, some studies found that the reduction of human bone MSCs can reduce the intestinal I/R injury mortality[33], and that human umbilical MSCs can provide an intestinal protective effect through nitric oxide dependent pathways[34]. Furthermore, studies have found that melatonin-supported adipose-derived MSCs[35], synergetic application of electroacupuncture and MSCs[36], HO-1-expressing BM-MSCs[37], IL-1 $\beta$-activated adiposederived MSCs [38], and IL-37 gene-modified MSCs also have a protective effect against intestinal I/R injury[39].

It has been confirmed that MSCs have therapeutic effects in animal models of intestinal I/R injury; however, the source of MSCs, administration dose, site of transplantation, and quality score in each study are very divergent that the overall therapeutic effect is difficult to evaluate, and there is no systematic review or meta-analysis on the effects of MSCs on therapy for intestinal I/R injury. To clarify the current situation and further studies on MSC therapy as a treatment for intestinal I/R injury, we will perform this systematic review and meta-analysis of all available experimental evidence to identify the efficacy of MSC-based therapies in animal models of intestinal I/R injury.

\section{OBJECTIVE}

The primary purpose of this systematic review is to examine the efficacy of MSCs in the treatment of intestinal I/R injury. Secondary specific aims are to determine the effects of different sources, administration doses, and administration sites of MSCs on intestinal I/R injury.

\section{Methods And Analysis}

This systematic review protocol was registered prospectively in the International Prospective Register of Systematic Reviews (PROSPERO) (registration number: CRD42021231826). The protocol follows the Preferred Reporting Items for Systematic Review and Meta-Analysis Protocols (PRISMA-P) statement[40].

\section{Eligibility criteria}

\section{Type of studies}

We will only include studies on controlled trials in animals. Reviews, editorials, study protocols, clinical guidelines, commentaries, and letters will be excluded.

\section{Type of participants}

An established animal disease model of intestinal I/R injury will be included. There are no restrictions on specific modelling methods, species and origin, age, and sex of the animals. No patient involved in this study.

\section{Type of interventions and comparators}


The intervention group will use MSCs as a therapy for the treatment of intestinal I/R injury, without restricting the type of MSCs, dose of cells, and site of transplantation. For comparisons, the control group can be another intervention except for MSCs or placebo.

\section{Types of outcome measures}

\section{Primary outcomes}

1. Degree of histopathologic changes, which will be graded semi-quantitatively using the histologic injury scale previously described by Chiu et al[41].

2. Mortality rate of rats

Secondary outcomes

1. Inflammatory cytokine: tumour necrosis factor a (TNF-a)

2. Marker of intestinal permeability: diamine oxidase (DAO)

Patient and Public Involvement

No patient involved in this study.

\section{Search methods for identification of studies}

A literature search of PubMed, Web of Science, Embase, Cochrane Library, Science Citation Index, China National Knowledge Information database, Wanfang Database, and Chinese Scientific and Technological Journal Database will be conducted from the inception to May 2021. The identified studies will not be constrained by publication date, language, or publication status. Furthermore, the following search strategy will be applied: (Mesenchymal stem cells, Bone Marrow Stromal Cells, Mesenchymal Progenitor Cells, Mesenchymal Stromal Cells) AND (intestinal ischemia-reperfusion injury, intestinal IR injury, IIR injury).

\section{Study selection}

Two authors will independently screen the titles and abstracts of studies searched by Rayyan [42] and will download the full text of any potential studies for further screening. Any discrepancies will be resolved by discussion; if needed, a third reviewer will be consulted. Figure 1 shows the flow chart of the study selection procedure according to the Preferred Reporting Items for Systematic Reviews and MetaAnalyses (PRISMA) statement.

\section{Data extraction and management}

Two authors will independently extract the data of interest. They will also resolve any discrepancies by having a discussion; if needed, a third reviewer will be consulted. The form will aim to capture information on study characteristics, including study design, sample size, experimental methods, intervention types, control, outcomes, funding, and so on. 


\section{Assessment of the risk of bias in individual studies}

Two authors will independently assess the risk of bias in each study using the SYRCLE tool[43]. This tool contains 10 entries related to selection bias, performance bias, detection bias, attrition bias, reporting bias, and other biases. A "yes" judgement indicates a low risk of bias, a "no" judgement indicates a high risk of bias, and an "unclear" judgment means that insufficient details have been reported to properly assess the risk of bias. Any conflicts will be resolved by discussion; if needed, a third reviewer will be consulted.

\section{Data synthesis}

Stata (version 12.0, Stata Corp, College Station, Texas, USA) will be used to analyse and pool the individual research results. For dichotomous outcomes, we will calculate a pooled estimate of risk ratios with $95 \%$ confidence intervals (Cls) using a random-effects model. Continuous outcomes will be analysed using the weighted mean difference with $95 \%$ Cls. For outcomes where different scales or different measurement methods have been used, the standardised mean difference will be applied.

\section{Assessment of heterogeneity}

We will estimate the degree of heterogeneity among studies using the Cochrane Q statistic and the $I^{2}$ statistic. Heterogeneity will be considered as small when $R^{2}<50 \%$ and substantial when $R^{2}>50 \%$. Additionally, we will perform a sensitivity analysis by excluding studies with a high risk of bias and will plan subgroup analyses based on different types and sources of MSCs, administration dose, and methods of MSC administration if sufficient studies are available.

\section{Publication bias}

A funnel plot will be generated to examine the publication bias if more than 10 studies are included to assess this endpoint.

\section{Ethics and dissemination}

This review does not require ethical approval as a systematic review of published studies because there is no direct involvement of human participants. We will publish our findings in a peer-reviewed scientific journal. The dataset will be made available based on reasonable requests from the researchers.

\section{Discussion}

Being the first meta-analysis to identify the efficacy of MSC-based therapies in animal models of intestinal I/R injury is one of the strengths of this systematic review. In addition, we will also determine the effects of different sources, administration doses, and administration sites of MSCs on intestinal I/R injury. Moreover, conducting meta-analyses and systematic reviews of the evidence related to this clinical question may lead to further research in this field and could provide the latest evidence for the treatment of intestinal I/R injury. 
We acknowledge the potential limitations of this study. First, we will include all types of MSCs. Although these inclusion criteria will contribute to increased pooled sample size, heterogeneity may be introduced. Nonetheless, we will conduct a subgroup analysis to evaluate the heterogeneity between different types of MSCs. Second, the strength of a systematic review and meta-analysis relies in part on the strength of available studies, and therefore may be limited due to the lack of randomised controlled trials in this area. Third, we also intend to perform subgroup analyses for the administration dose and site of MSCs. However, this may reduce the statistical power of data analysis.

\section{Declarations}

\section{Authors' contributions}

LYF, GL and ZGR devised the study. ZGR drafted the protocol, and all authors provided suggestions regarding manuscript revisions. The search strategy developed by ZGR will be performed by ZGR and LZZ, who will also independently screen the potential studies. ZGR, LZZ, LDS, ZZY, and SSH will independently extract data from the included studies, assess the risk of bias, and complete the data synthesis. ZGR and GL will arbitrate in cases of disagreement. All authors approved the publication of this protocol.

\section{Acknowledgements}

We would like to thank Qi Wang (MPH candidate, Lanzhou University) and Ting Zhang (PhD candidate, Traditional Chinese Medicine of Tianjin University) for providing suggestions regarding manuscript revisions.

\section{Funding}

This work is supported by the projects of the National Natural Science Foundation of China (No. 81960345).

\section{Competing interest statement}

No conflicts of interest to declare by any of the authors.

\section{References}

1. Turan I, Sayan Ozacmak H, Ozacmak VH et al. The effects of S-nitrosoglutathione on intestinal ischemia reperfusion injury and acute lung injury in rats: roles of oxidative stress and NF-kB. Tissue Cell 2018;52:35-41.

2. Grootjans J, Lenaerts K, Buurman WA et al. Life and death at the mucosal-luminal interface: new perspectives on human intestinal ischemia-reperfusion. World J Gastroenterol: English Edition 2016;22:2760-70. 
3. Liu J, Chen T, Lei P et al. Exosomes released by bone marrow mesenchymal stem cells attenuate lung injury induced by intestinal ischemia reperfusion via the TLR4/NF-KB pathway. Int J Med Sci 2019;16:1238-44.

4. Liu J, Pan G, Liang T et al. HGF/c-Met signaling mediated mesenchymal stem cell-induced liver recovery in intestinal ischemia reperfusion model. Int J Med Sci 2014;11:626-33.

5. Matsuda A, Yang WL, Jacob A, et al. FK866, a visfatin inhibitor, protects against acute lung injury after intestinal ischemia-reperfusion in mice via NF-kB pathway. Ann Surg 2014;259:1007-17.

6. Fishman JE, Sheth SU, Levy G, et al. Intraluminal nonbacterial intestinal components control gut and lung injury after trauma hemorrhagic shock. Ann Surg 2014;260:1112-20.

7. Oldenburg WA, Lau LL, Rodenberg TJ et al. Acute mesenteric ischemia: A clinical review. Arch Intern Med 2004;164:1054-62.

8. Abu-Elmagd K, Reyes J, Bond $\mathrm{G}$ et al. Clinical intestinal transplantation: a decade of experience at a single center. Ann Surg 2001;234:404-16; discussion 416.

9. Hu C, Zhao L, Wu D et al. Modulating autophagy in mesenchymal stem cells effectively protects against hypoxia- or ischemia-induced injury. Stem Cell Res Ther 2019;10:120.

10. Gomez L, Li B, Mewton N, et al. Inhibition of mitochondrial permeability transition pore opening: translation to patients. Cardiovasc Res 2009;83:226-33.

11. Fatih A, Dominique PV, et al. Bridging innate immunity and myocardial ischemia/reperfusion injury: the search for therapeutic targets. Curr Pharm Des 2008.

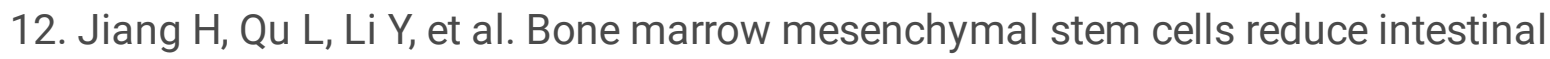
ischemia/reperfusion injuries in rats. J Surg Res 2011;168:127-34.

13. Khanna A, Rossman J, Caty MG et al. Beneficial effects of intraluminal nitroglycerin in intestinal ischemia-reperfusion injury in rats. J Surg Res 2003;114:15-24.

14. Yufang $S$, Yu W, Qing L, et al. Immunoregulatory mechanisms of mesenchymal stem and stromal cells in inflammatory diseases. Nat Rev Nephrol 2018; 14:493-507.

15. Kim KH, Blasco-Morente G, Cuende $\mathrm{N}$ et al. Mesenchymal stromal cells: properties and role in management of cutaneous diseases. J Eur Acad Dermatol Venereol 2017;31:414-23.

16. Yang XF, Chen T, Ren LW et al. Immunogenicity of insulin-producing cells derived from human umbilical cord mesenchymal stem cells. Exp Ther Med 2017;13:1456-64.

17. Le Blanc K, Rasmusson I, Sundberg $B_{\text {, }}$ et al. Treatment of severe acute graft-versus-host disease with third party haploidentical mesenchymal stem cells. Lancet 2004; 363:1439-41.

18. Mushahary D, Spittler A, Kasper $C$ et al. Isolation, cultivation, and characterization of human mesenchymal stem cells. Cytometry A 2018;93:19-31.

19. Zuk PA, Zhu M, Ashjian P et al. Human adipose tissue is a source of multipotent stem cells. Mol Biol Cell 2003;13:4279-95.

20. da Silva Meirelles L, Chagastelles PC, Nardi NB. Mesenchymal stem cells reside in virtually all postnatal organs and tissues. J Cell Sci 2006;119:2204-13. 
21. Yan Q, Ruan JW, Ding Y et al. Electro-acupuncture promotes differentiation of mesenchymal stem cells, regeneration of nerve fibers and partial functional recovery after spinal cord injury. Exp Toxicol Pathol 2011;63:151-6.

22. Wong CY, Cheong SK, Mok PL et al. Differentiation of human mesenchymal stem cells into mesangial cells in post-glomerular injury murine model. Pathology 2008;40:52-7.

23. Zarjou A, Kim J, Traylor AM et al. Paracrine effects of mesenchymal stem cells in cisplatin-induced renal injury require heme oxygenase-1. Am J Physiol Ren Physiol 2010;300:F254-62.

24. Murphy MB, Moncivais K, Caplan Al. Mesenchymal stem cells: environmentally responsive therapeutics for regenerative medicine. Exp Mol Med 2013;45:e54.

25. Shafei AE, Ali MA, Ghanem HG, et al. Mesenchymal stem cell therapy: A promising cell-based therapy for treatment of myocardial infarction. J Gene Med 2017;19(12): e2995.

26. Liu J, Huang Y, He J et al. Olfactory mucosa mesenchymal stem cells ameliorate cerebral ischemic/reperfusion injury through modulation of UBIAD1 expression. Front Cell Neurosci 2020;14:580206.

27. Liau LL, Looi QH, Chia WC et al. Treatment of spinal cord injury with mesenchymal stem cells. Cell Biosci 2020;10:112.

28. Zheng J, Chen L, Lu T, et al. MSCs ameliorate hepatocellular apoptosis mediated by PINK1dependent mitophagy in liver ischemia/reperfusion injury through AMPKalpha activation. Cell Death Dis 2020;11:256.

29. Missoum A. Recent updates on mesenchymal stem cell based therapy for acute renal failure. Curr Urol 2020;13:189-99.

30. Shen ZY, Zhang J, Song HL et al. Bone-marrow mesenchymal stem cells reduce rat intestinal ischemia-reperfusion injury, ZO-1 downregulation and tight junction disruption via a TNF-alpharegulated mechanism. World J Gastroenterol 2013;19:3583-95.

31. Jiang H, Qu L, Dou R et al. Potential role of mesenchymal stem cells in alleviating intestinal ischemia/reperfusion impairment. PLOS ONE 2013;8:e74468.

32. Inan M, Bakar E, Cerkezkayabekir A, et al. Mesenchymal stem cells increase antioxidant capacity in intestinal ischemia/reperfusion damage. J Pediatr Surg 2017;52:1196-206.

33. Markel TA, Crafts TD, Jensen AR et al. Human mesenchymal stromal cells decrease mortality after intestinal ischemia and reperfusion injury. J Surg Res 2015;199:56-66.

34. Jensen AR, Drucker NA, Ferkowicz MJ et al. Umbilical mesenchymal stromal cells provide intestinal protection through nitric oxide dependent pathways. J Surg Res 2018;224:148-55.

35. Chang CL, Sung PH, Sun CK, et al. Protective effect of melatonin-supported adipose-derived mesenchymal stem cells against small bowel ischemia-reperfusion injury in rat. $J$ Pineal Res 2015;59:206-20.

36. Geng Y, Chen D, Zhou J, et al. Synergistic effects of electroacupuncture and mesenchymal stem cells on intestinal ischemia/reperfusion injury in rats. Inflammation 2016;39:1414-20. 
37. Yan XT, Cheng XL, He XH et al. The HO-1-expressing bone mesenchymal stem cells protects intestine from ischemia and reperfusion injury. BMC Gastroenterol 2019;19:124.

38. Liu L, He YR, Liu SJ, et al. Enhanced effect of IL-1beta-activated adipose-derived MSCs (ADMSCs) on repair of intestinal ischemia-reperfusion injury via COX-2-PGE2 signaling. Stem Cells Int 2020;2020:2803747.

39. Kong D, Hu Y, Li X, et al. IL-37 gene modification enhances the protective effects of mesenchymal stromal cells on intestinal ischemia reperfusion injury. Stem Cells Int 2020;2020:8883636.

40. Shamseer L, Moher D, Clarke M, et al. Preferred reporting items for systematic review and metaanalysis protocols (PRISMA-P) 2015: elaboration and explanation. BMJ 2015;350:g7647

41. Chiu CJ, Mcardle AH, Brown R et al. Intestinal mucosal lesion in low-flow states: I. A morphological, hemodynamic, and metabolic reappraisal. Arch Surg 1970;101:478-83.

42. Ouzzani M, Hammady H, Fedorowicz Z, et al. Rayyan-a web and mobile app for systematic reviews. Systematic Reviews 2016;5:210.

43. Hooijmans CR, Rovers MM, de Vries RB, et al. SYRCLE's risk of bias tool for animal studies. BMC Med Res Methodo/ 2014;14:43.

\section{Figures}




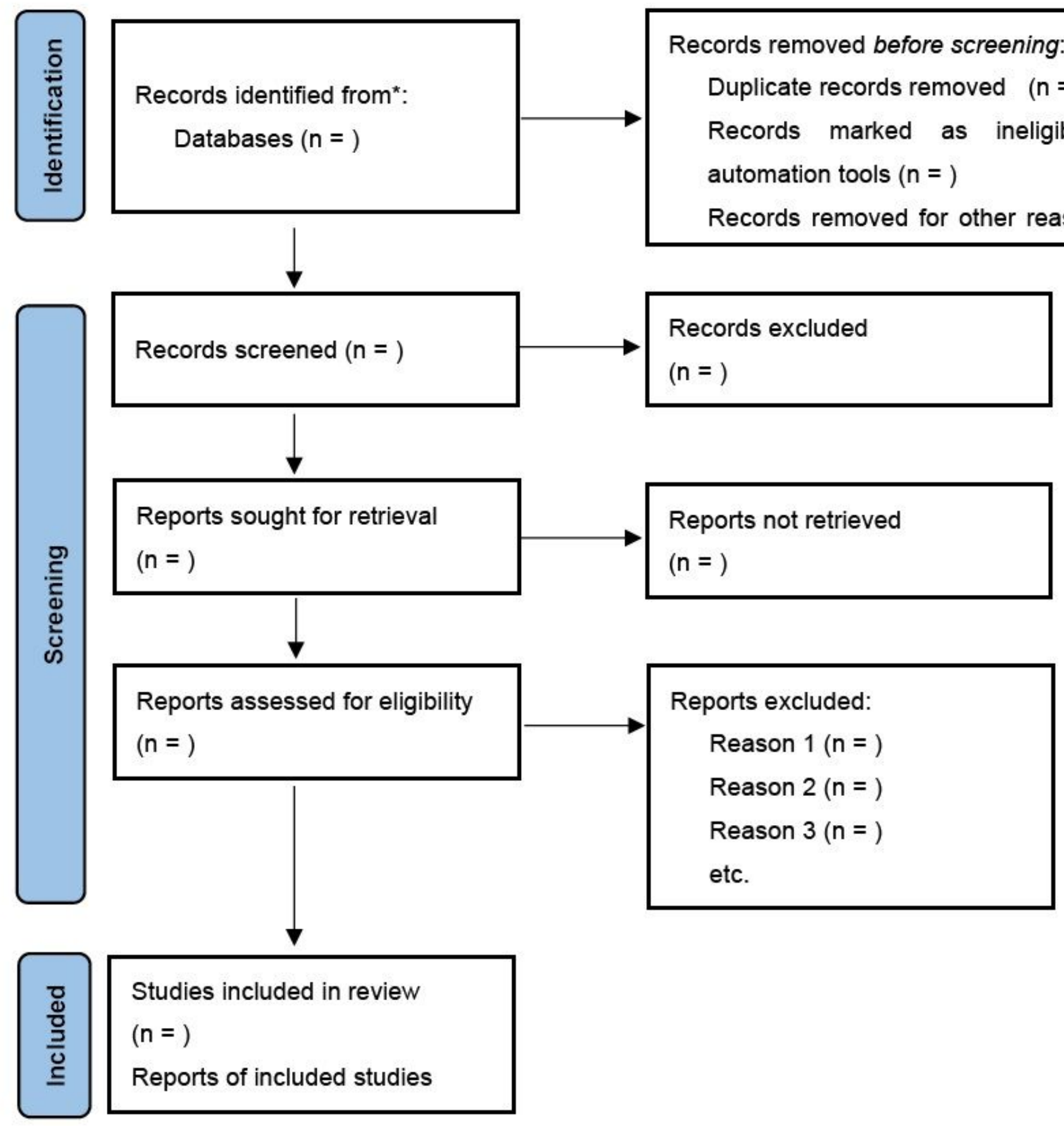

Figure 1

Flow chart of the study selection procedure. The flow chart of the study selection procedure is based on the Preferred Reporting Items for Systematic Reviews and Meta-Analyses (PRISMA) Statement.

\section{Supplementary Files}

This is a list of supplementary files associated with this preprint. Click to download.

- PRISMAPchecklist.doc 